\title{
Individual Differences in Children's Early Strategy Behavior in Arithmetic Tasks
}

\author{
Sven Lindberg ${ }^{1,2}$, Janosch Linkersdörfer ${ }^{1,2}$, Martin Lehmann ${ }^{1,2}$, Marcus Hasselhorn ${ }^{1,2,3}$ \& Jan Lonnemann ${ }^{1,2}$ \\ ${ }^{1}$ German Institute for International Educational Research (DIPF), Frankfurt, Germany \\ ${ }^{2}$ Center for Individual Development and Adaptive Education of Children at Risk (IDeA), Frankfurt, Germany \\ ${ }^{3}$ Institute for Psychology, Goethe-University, Frankfurt, Germany \\ Correspondence: Sven Lindberg, IDeA, Schloßstr. 29, 60486 Frankfurt am Main, Germany. Tel: \\ 49-069-2470-8216. E-mail: s.lindberg@idea-frankfurt.eu
}

Received: February 9, 2013

Accepted: March 27, 2013 Online Published: April 11, 2013

doi:10.5539/jedp.v3n1p192

URL: http://dx.doi.org/10.5539/jedp.v3n1p192

\begin{abstract}
As demonstrated by the Overlapping Waves Model (Siegler, 1996), children's strategy use in arithmetic tasks is variable, adaptive, and changes gradually with age and experience. In this study, first grade elementary school children $(\mathrm{n}=73)$, who scored high, middle, or low in a standardized scholastic mathematic achievement test, were confronted with different arithmetic tasks (simple addition, e.g., $3+2$, simple subtraction, e.g., $7-2$, and more advanced addition, e.g., $7+9$ ) to evoke different calculation strategies. Video analysis and children's self-report were used to identify individual strategy behavior. In accordance with the Overlapping Waves Model, children in all achievement groups showed variable and multiple strategy usage and adapted their behavior to the tasks of the different categories. We demonstrated that not only low achievers differed from normal achievers but also that high achievers exhibited a unique pattern of strategy behavior in early mathematics.
\end{abstract}

Keywords: elementary school, math strategies, math achievement, overlapping waves model

\section{Introduction}

Children's development of thinking can be described as variable, adaptive, and gradually changing over time. An individual has at any moment the opportunity to use several approaches to solve a specific problem. The occurrences, increase, decrease, and coexistence of specific strategies can be thereby imagined as overlapping waves in a diagram representing the probability of their application to a certain task (Overlapping Waves Model - OWM; Siegler, 1996). Research in the mathematical domain has examined the strategy behavior of typical developing children and adults according to the OWM in detail. Results indicate that while individuals exhibit a wide range of different strategies, less effective and time-consuming strategies (such as counting) will be initially replaced by more elaborated and finally by retrieval-based strategies in the course of development (Zbrodoff \& Logan, 2005).

Before entering school, children start to approach calculation by using guessing and estimation strategies (Jordan, Kaplan, Ramineni, \& Locuniak, 2008). With the beginning of instructional education, they start with the addition and subtraction of single digits by using finger or verbal counting strategies (Geary, Hoard, Nugent, \& Byrd Craven, 2007). In the course of time, children build up factual knowledge due to repeated calculation experiences. Thereby, a linkage will be established between a problem and its respective answer stored in long-term memory that enables the children to use more advanced or even retrieval based strategies (Siegler \& Shrager, 1984). In this regard, the individually acquired knowledge base, which contains experiences with specific problems, is decisive for the selection and application of a specific strategy (Mabbott \& Bisanz, 2003). Although a child might use direct fact retrieval in most simple addition tasks, he or she may not use retrieval in all these tasks. If a problem is unfamiliar, i.e., if there is no entry for the respective task's result in long-term memory, or if the child is unsure about the retrieved result, he or she may choose an alternative strategy that is more likely to produce a correct answer (Siegler, 1988). Other findings emphasize that strategy selection is important for the development of mathematical achievement and that strategy behavior changes with growing experience in mathematical learning (Lemaire \& Siegler, 1995; Imbo \& Vandierendonck, 2007). Moreover, variability of strategy behavior seems to be important for the learning process itself and for later success in more 
advanced arithmetic (Siegler, 2007).

Several studies investigated the typical development of children's mathematical strategy behavior. A longitudinal study (Lemaire \& Siegler, 1995) showed that normal achieving children at the beginning of $2^{\text {nd }}$ year elementary school applied retrieval (38\%), counting strategies (30\%), and saying "I don't know" (32\%) equally often when solving multiplication problems. At the end of $2^{\text {nd }}$ grade, this pattern completely changed caused by a massive shift to direct retrieval of arithmetic facts (92\% retrieval, $6 \%$ counting strategies, and $2 \%$ "I don't know"). For single digit addition tasks (Imbo \& Vandierendonck, 2007), it was demonstrated that normal achieving fourth grade elementary school children used retrieval (46\%) and elaborated strategies $(41 \%)$ equally often whereas fifth and sixth graders used retrieval $(67 \%$ and $60 \%)$ more often than elaborated strategies $(15 \%$ and $22 \%)$. Less elaborated strategies such as counting were used rather infrequently in all grades $\left(4^{\text {th }} 12 \%, 5^{\text {th }} 18 \%\right.$ and $\left.6^{\text {th }} 18 \%\right)$. Overall, the applied strategies mainly lead to the correct answers and were therefore selected adequately. Comparable findings were obtained for more complex arithmetic problem solving (e.g., $24+53$ ) comparing fifth and seventh graders to adults (Lemaire \& Calliès, 2009).

Children with mathematical difficulty (MD) seem, at first view, not to differ from normal developing children regarding their repertory of available strategies. Upon closer examination, however, it becomes apparent that children with MD predominantly use less proficient strategies and relapse over and over to using counting procedures (Geary \& Hoard, 2005). Moreover, in these children the usage of more elaborated strategies such as decomposition and retrieval seems to be reduced and, when applied, more error-prone (Geary et al., 2009). Surprisingly, seven-year-old children with MD seem to exhibit counting and retrieval strategies to a comparable extent, while normal achieving children at the same age nearly completely rely on counting strategies. In terms of accuracy, however, it becomes evident that MD children use their strategies less effectively, resulting in high error rates, whereas normal achieving children are almost always able to correctly solve the tasks with their chosen strategy (Geary, Hamson, \& Hoard, 2000). Therefore, children with MD seem to have problems to use their available strategies adequately and, for instance, use fact retrieval although they are not sure if the retrieved result is really the correct answer. At the age of eight, a change in children's mathematical strategy behavior is observable. While normal achieving children use more and more elaborated and retrieval based strategies, children with MD turn back to the usage of counting orientated strategies (Geary et al., 2000). At least MD children are able to use the counting strategies considerable correctly. That gain is, however, accompanied by a neglect of more elaborated strategy usage. In the course of elementary school, children with MD seem to use their strategy repertory only marginally adaptive and to apply less elaborated strategies more frequently than retrieval and elaborated strategies (Geary, Brown, \& Samaranayake, 1991; Ostad, 1997).

As described above, research on (elementary school) children's mathematical strategy behavior revealed explicit differences between normal achieving children and children with MD. However, the full range of mathematical ability levels in children includes, besides the impaired and normal development, also achievement that is above average. Therefore, the current study aimed at investigating the use of various strategies in different arithmetic tasks in first grade elementary school children, who were identified as low, normal or high achieving in mathematics based on their performance in a standardized scholastic test. According to previous findings, we expected that all children would use various strategies in the different arithmetic tasks. Normal and high achieving children should apply more advanced strategies and use their selected strategies more adaptively and effectively than the low achieving children. Finally, a main goal of the study was to provide answers to the question to what extent high achieving children differ from the other two groups regarding their strategy behavior and if they display a unique strategy pattern.

\section{Method}

\subsection{Participants}

A total of 73 first grade (35 female; mean age 7.2) students were recruited from German elementary schools in the area of Frankfurt. Children's mathematical school achievement was measured by the standardized German test for the availability of basic facts in arithmetics (DIRG, Grube, Weberschock, Stuff, \& Hasselhorn, 2010). Based on their DIRG scores, children were assigned to three groups: Low achievement (LA) $=$ scores below the 33th percentile, normal achievement $(\mathrm{NA})=$ scores between the 33th and the 66th percentile and high achievement $(\mathrm{HA})=$ scores above the 66th percentile. Parental informed and written consent was obtained for each child.

\subsection{Materials}

The children were presented with a set of 42 arithmetic tasks, which were presented individually on sheets of a pad. The set consisted of 18 simple addition (e.g., $3+2$ ) and 12 simple subtraction (e.g., $4-2$ ) tasks in the 
number range up to ten and 12 advanced addition (e.g., $8+7$ ) tasks in the number range up to twenty.

\subsection{Procedure}

Participating children were tested individually by trained student assistants using a microgenetic trial-by-trial procedure during which a task related question on the solution was asked after each presented problem (Miller, 2000; Siegler, 2006). As an introduction, children were presented with an example to demonstrate the procedure. The applied proceeding was based on the original instructions used in various studies that examined strategy behavior (e.g., Rittle-johnson \& Siegler, 1999; Siegler, 1988). After each task, the children were asked "How did you solve this problem?". Additionally, the complete session was videotaped to record children's self-report and their overt strategy behavior.

\subsection{Strategy Coding}

Children's videotaped overt and self-reported strategy behavior was coded in a subsequent analysis. According to previous research on strategy development (Lemaire \& Siegler, 1995; Canobi, Reeve, \& Pattison, 2003; Canobi, 2004; Lindberg et al., 2011) categories were predefined resulting in the following categories (a) retrieval (direct answer), (b) elaborated strategies (decomposition), (c) less elaborated strategies (counting) and (d) unspecific strategies (e.g., guessing and unclassifiable behavior or self-report) to ease terms of statistical analyses. Strategies were coded according to the following rules: (1) corresponding observed behavior and self-report = direct coding, (2) observed behavior and self-report divergent = coding followed the observed behavior, (3) no observable strategy behavior and self-report = coding followed children's self-report.

\section{Results}

\subsection{Variability}

To verify our assumption that all children would use various strategies while solving the different arithmetic tasks, we examined the frequencies of each strategy category for the three groups in the simple addition, simple subtraction, and advanced addition problems.

Firstly, none of the children in the LA group used only one strategy category in the simple addition tasks. Only $8.7 \%$ (two children) used two strategy categories, $47.8 \%$ used three strategy categories, and $43.5 \%$ used four strategy categories. When solving simple subtraction tasks, $4.3 \%$ (one child) used only one strategy category, $52.2 \%$ used two strategy categories, $39.1 \%$ used three strategy categories, and $4.3 \%$ used four strategy categories. In the advanced addition tasks, $13 \%$ (three children) used only one strategy category, $43.5 \%$ used two strategy categories, $21.7 \%$ used three strategy categories, and $21.7 \%$ used four strategy categories.

Secondly, none of the children in the NA group used only one strategy category in the simple addition tasks. However, $23.1 \%$ used two strategy categories, $46.2 \%$ used three strategy categories, and $30.8 \%$ used four strategy categories. When solving simple subtraction tasks, $3.8 \%$ (one child) used only one strategy category, $30.8 \%$ used two strategy categories, $61.5 \%$ used three strategy categories, and 3.8\% used four strategy categories. In the advanced addition tasks, $15.4 \%$ (four children) used only one strategy category, $30.8 \%$ used two strategy categories, $38.5 \%$ used three strategy categories, and again $15.4 \%$ used four strategy categories.

Thirdly, none of the children in the HA group used only one strategy category in the simple addition tasks. Instead, $54.2 \%$ used two strategy categories, $25 \%$ used three strategy categories, and $20.8 \%$ used four strategy categories. When solving simple subtraction tasks $12.5 \%$ (three children) used only one strategy category, $50 \%$ used two strategy categories, $37.5 \%$ used three strategy categories, and none used four strategy categories. In the advanced addition tasks, $12,5 \%$ (three children) used only one strategy category, $45,8 \%$ used two strategy categories, 33,3\% used three strategy categories, and 8,3\% (two children) used four strategy categories.

\subsection{Strategy Use and Adaptive Behavior}

We hypothesized that children in the NA and HA groups would apply more advanced strategies and would use their selected strategies more adaptively and effectively than the children in the LA group. As expected, children's strategy behavior varied in regard to the presented tasks (indicating that the tasks simple addition, simple subtraction, and advanced addition represent an ascending order in terms of difficulty). Table 1 displays the means of percentages of strategy use for children of the three achievement groups as a function of the different arithmetic tasks. 
Table 1. Means (and standard deviations) of percentages strategy usage as a function of arithmetic task, achievement group, and strategy

\begin{tabular}{cllll}
\hline & & \multicolumn{3}{l}{ Strategy } \\
\cline { 2 - 5 } & retrieval & elaborated & less elaborated & unspecific \\
\hline Low achiever & & & \\
simple addition & $38.8(22.1)$ & $5.5(6.4)$ & $22.4(21.9)$ & $32.6(19.2)$ \\
simple subtraction & $29.7(24.7)$ & $0.3(1.7)$ & $19.9(21.4)$ & $50(27.9)$ \\
advanced addition & $16.6(23.5)$ & $10.8(16.5)$ & $39.4(36.6)$ & $29.3(28.9)$ \\
Normal achiever & & & & \\
simple addition & $51.7(24.3)$ & $2.9(4.5)$ & $21.1(23.8)$ & $24.1(19.5)$ \\
simple subtraction & $45.1(27.3)$ & $1.6(4)$ & $16(23)$ & $37.1(22.6)$ \\
advanced addition & $32.3(31.8)$ & $16.6(25.7)$ & $25.6(34.2)$ & $22.4(28.7)$ \\
High achiever & & & & \\
simple addition & $74(18.1)$ & $2.7(5.4)$ & $8.1(13)$ & $15(11.5)$ \\
simple subtraction & $67(23.3)$ & $1(5.1)$ & $7.9(13.1)$ & $23.9(20)$ \\
advanced addition & $50.3(36.4)$ & $3.1(8.7)$ & $16.6(24.8)$ & $29.8(28.3)$ \\
\hline
\end{tabular}

Generally, children of the HA group used predominantly retrieval and outperformed the other achievement groups considerably in this respect. Moreover, also NA children used more retrieval than the LA children. In contrast, both LA and NA children used less elaborated (counting based) strategies to a comparable amount. Unspecific strategy behavior was most prevalent in LA children and elaborated strategies were rather rarely used in all groups.

To compare the frequencies of the applied strategy categories statistically we conducted a 3 (group) $x 3$ (arithmetic task) x 4 (strategy type) repeated measures analysis of variance (ANOVA). Since this ANOVA uses ipsative measures on the first two factors, the degrees of freedom are adjusted by Box's epsilon to control Type I error rates (see Greer \& Dunlap, 1997). No main effects for arithmetic task and no main effect for group can be reported since for all tasks and in all groups strategy behavior was present. A significant main effect of strategy type was found, $F(3,210)=48.67, p<.001, \eta^{2}=0.41$. This main effect was qualified by significant two-way interactions between strategy type and group, $F(6,210)=8.22, p<.001, \eta^{2}=0.19$ and strategy type and arithmetic task, $F(6,420)=15.96, p<.001, \eta^{2}=0.18$. Additionally, the three-way interaction arithmetic task, strategy type, and group, $F(12,420)=1.87, p<.05, \eta^{2}=0.05$, was also significant. As can be seen in Table 1 , it seems that the strategy behavior of the three achievement groups differs across the three arithmetic tasks. LSD post-hoc comparisons were conducted to analyze strategy-specific differences within and between the three achievement groups:

LA children used predominantly retrieval and unspecific strategies, on a comparable level, when solving simple addition tasks. Less elaborated strategies were on a similar level with unspecific strategies and both were significantly more often used than elaborated strategies. In the simple subtraction tasks, LA children used mostly unspecific strategies, followed by retrieval as the second most common, but significantly less frequently used, strategy. The frequency of less elaborated strategies did not differ significantly from the frequency of retrieval, but was significantly higher than the frequency of elaborated strategies, which were almost never used. For advanced addition, less elaborated and unspecific strategies did not differ significantly and were used most often. Retrieval and elaborated strategies differ not from each other and were used only seldom. 
NA children used retrieval significantly more frequently than other strategies when solving simple addition tasks. Hereafter, they applied unspecific strategies and retrieval to a comparable degree, whereas elaborated strategies were only seldomly applied. For simple subtraction, retrieval and unspecific strategies were used equally often and significantly more often than less elaborated strategies. Again, elaborated strategies were nearly not present. Retrieval, less elaborated, and unspecific strategies were comparably often used to solve advanced addition tasks and their frequencies did not differ significantly from each other. Also, the less prevalent elaborated strategies only differed significantly from the usage of retrieval but not from the less elaborated and unspecific strategies.

Across all tasks (simple addition, subtraction, and advanced addition), HA children used retrieval significantly more often than any other strategy. With increasing level of task difficulty, unspecific strategies were applied more often as the second most frequent alternative. For simple and advanced addition, however, less elaborated and unspecific strategies did not differ significantly. Noticeably, elaborated strategies were nearly not applied at all.

The overall comparison across the three achievement groups revealed that HA children clearly outperformed the children of the two other groups in regard to the frequency of the use of retrieval, independent from the type of the arithmetic task. Moreover, also NA children used significantly more retrieval than the children of the LA group in all arithmetic tasks. Unspecific strategies were significantly less frequently used by HA children in simple addition and subtraction tasks, but the usage was comparable across all groups in advanced addition tasks. LA and NA children applied less elaborated strategies comparably often, whereas HA children used these strategies significantly less often in simple addition and subtraction tasks. Elaborated strategies were used very seldom. Only for advanced addition task, LA and NA children used that strategy category comparably often, with HA children using it significantly less often than the NA group.

\subsection{Strategy Effectiveness}

We assumed that children in the NA and HA groups would use their chosen strategies more effectively than children in the LA group. To analyze children's effectiveness, we looked at the frequency of errors that were observed in the three groups across the simple addition, simple subtraction, and advanced addition problems.

Firstly, when solving simple addition tasks, $56.6 \%$ of the children in the LA group made no mistakes. However, $34.8 \%$ made one mistake whereas two and three mistakes were each made by $4.3 \%$ (one child). In the simple subtraction tasks, $52.2 \%$ of the children made no mistakes, $34.8 \%$ one error, and two, seven, and eight mistakes were each made by $4.3 \%$. For advanced addition, it was found that $43.5 \%$ made no, $8.7 \%$ one, $17.4 \%$ two, $4.3 \%$ four, $8.7 \%$ five, $4.3 \%$ six, $4.3 \%$ seven, and $8.7 \%$ eight mistakes.

Secondly, when solving simple addition tasks, $84.6 \%$ of the children in the NA group made no and $15.4 \%$ one mistake. For simple subtraction tasks, it was found that $76.9 \%$ made no, $11.5 \%$ one, $3.8 \%$ (one child) four, and 7.7\% (two children) made 12 mistakes (children accidently ignored the minus sign). In advanced addition, it was observed that $50 \%$ made no, $15.4 \%$ one, $19.2 \%$ two, $3.8 \%$ (one child) three, $3.8 \%$ four, $3.8 \%$ six, and $3.8 \%$ seven mistakes.

Thirdly, when solving simple addition tasks $100 \%$ of the children in the HA group made no mistakes. In simple subtraction $95.8 \%$ made no and $4.2 \%$ (one child) only one mistake. For advanced addition, it was found that $79.2 \%$ made no, $16.2 \%$ one, and $4.2 \%$ (one child) four mistakes.

For a closer examination of the observed mistakes in regard to the applied strategies, we computed the percentage of errors as a function of arithmetic task, achievement group, and strategy (see Table 2). To compare the frequency of the occurred errors across the different tasks and for the used strategies between the three groups statistically, we conducted a 3 (group) x 3 (arithmetic task) x 4 (strategy type) repeated measures analysis of variance (ANOVA). 
Table 2. Means (and standard deviations) of the percentage of errors as a function of arithmetic task, achievement group, and strategy

\begin{tabular}{lllll}
\hline & \multicolumn{3}{c}{ Strategy } \\
\cline { 2 - 5 } & retrieval & elaborated & less elaborated & unspecific \\
\hline Low achiever & & & \\
simple addition & $0.2(1.5)$ & $0(0)$ & $0.9(2.7)$ & $1.9(3.1)$ \\
simple subtraction & $1.4(5.4)$ & $0(0)$ & $1.8(4.3)$ & $5.7(14)$ \\
advanced addition & $0(0)$ & $3.2(8.2)$ & $9.4(16.9)$ & $6.5(15.2)$ \\
Normal achiever & & & & \\
simple addition & $0.2(1)$ & $0(0)$ & $0.2(1)$ & $0.4(1.5)$ \\
simple subtraction & $0.6(2.2)$ & $0(0)$ & $1.9(5.9)$ & $7.3(21.1)$ \\
advanced addition & $2.8(8.4)$ & $2.8(5.7)$ & $2.5(7.7)$ & $2.5(5.1)$ \\
High achiever & & & & $0(0)$ \\
simple addition & $0(0)$ & $0(0)$ & $0(0)$ & $0(0)$ \\
simple subtraction & $0(0)$ & $0(0)$ & $0.3(1.7)$ & $1.3(6.8)$ \\
advanced addition & $0.6(2.3)$ & $0(0)$ & $0.6(2.3)$ & \\
\hline
\end{tabular}

Significant main effects for arithmetic task $F(2,140)=8.13, p<.001, \eta^{2}=0.10$ and for group $F(2,70)=6.85, p$ $<.005, \eta^{2}=0.16$ can be reported indicating that differences in the occurrence of errors are present in principle. Moreover, a significant main effect of strategy type was found, $F(3,210)=5.98, p<.001, \eta^{2}=0.07$. The corresponding two-way interactions between strategy type and group and strategy type and arithmetic task were not significant. However, the three-way interaction arithmetic task, strategy type and group, $F(12,420)=1.71, p$ $=.06, \eta^{2}=0.04$, was marginally significant on the level of $.10(p=.062)$. As can be seen in Table 2 , children in all groups made generally very few mistakes. Children of the HA clearly outperformed the other two groups by using all applied strategies most effectively. LA and NA children both appear to be more error-prone when using unspecific strategies in simple subtraction. Moreover, LA and NA children significantly less effectively use their strategies in advanced addition. Especially, children of the LA group made significantly more mistakes when applying less elaborated strategies.

\section{Discussion}

The aim of the current study was to examine the mathematical strategy behavior patterns of low, normal, and high achieving children in the first grade of elementary school. Based on the results of previous research with LA and NA children (Geary et al., 2000; Geary \& Hoard, 2005; Geary et al., 2009; Wylie, Jordan, \& Mulhern, 2012), we expected a different strategy pattern for the LA compared to the NA group. Since so far the strategy behavior of high achieving children was not considered explicitly, we were interested to compare the strategy use of HA children in early arithmetic to the other two groups.

First of all, we provided additional evidence to the assumptions of the OWA (Siegler, 1996), by demonstrating that children of all groups used multiple strategies and applied them adaptively across arithmetic tasks with different difficulty levels. Moreover, we were able to compare the strategy pattern of first grade LA and NA children and relate the results to previous findings. In this regard, it becomes apparent that, when focusing only at the typical development (NA children), the children in our sample showed already a substantial amount of retrieval use across the different arithmetic tasks. In line with other findings (Lemaire \& Siegler, 1995; Imbo \& Vandierendonck, 2007), also less elaborated (counting based) and unspecific strategies were applied relatively 
often. However, when contrasting our results with the findings of a study that compared LA and NA children in the age of 7 and 8 years (Geary et al., 2000), several differences were distinguishable. To examine children's effectiveness and adaptive behavior in their strategy use, we presented arithmetic tasks with increasing difficulty level. Therefore, we were able to analyze the frequencies with which children applied different strategies and committed errors across the different tasks. We found that NA children used significantly more retrieval than LA children across all arithmetic tasks, but used elaborated, less elaborated and unspecific strategies comparably frequent. In terms of effectiveness, LA children made generally more errors, but in advanced addition tasks NA children exhibited a comparable error rate. When focusing only on the advanced addition tasks, NA children showed a comparable frequency of retrieval use, less elaborated and unspecific strategies, whereas the LA children relied only on the two last-mentioned strategies indicating a reduction in retrieval usage. Although in simple subtraction LA children showed already a certain amount of retrieval they still used more unspecific strategies. Nevertheless, despite the stated differences, especially the results of the more advanced arithmetic tasks seem to be again in accordance with the above-mentioned findings from Geary et al. (2000). Thus, we could generally confirm our assumptions concerning the LA and NA children.

With respect to our explorative research question to what extent high achieving children differ from the other two groups regarding their strategy behavior and if they display a unique strategy pattern, we compared the mathematical strategy behavior of HA children to the strategy behavior of the two other groups. Our results indicate that HA children use retrieval as their predominant strategy across all arithmetic tasks and seem to apply it very effectively. Moreover, HA children, compared to both, LA and NA children, made nearly no mistakes regardless of which strategy they used. Moreover, HA children used elaborated and less elaborated strategies only to a small degree, whereas the usage of unspecific strategies was comparably prevalent in the group of NA children. The most striking result regarding HA children emerges when interpreting our findings in the context of the study of Imbo and Vandierendonck (2007). They investigated the strategy behavior of $4^{\text {th }}, 5^{\text {th }}$, and $6^{\text {th }}$ graders in single digit addition (with comparable tasks as in our simple and advanced addition tasks). As mentioned in the introduction, they found that normal achieving forth grade elementary school children used retrieval (46\%) and elaborated strategies (41\%) equally often, whereas fifth and sixth graders most frequently used retrieval $(67 \%$ and $60 \%)$ and less often elaborated strategies (15\% and 22\%). Less elaborated strategies such as counting were used rather infrequently in all grades $\left(4^{\text {th }} 12 \%, 5^{\text {th }} 18 \%\right.$ and $\left.6^{\text {th }} 18 \%\right)$ and the applied strategies generally lead to the correct results. In our sample, the HA group used already $74 \%$ retrieval in addition tasks in the number range up to ten (simple addition) and 50.3\% retrieval in addition tasks in the number range above ten (advanced addition) and therefore already exhibit a strategy pattern that is comparable to considerably older children.

As can be seen in Table 1, children in all achievement groups applied a considerable amount of unspecific strategies and only very few elaborated strategies. Since in our sample the usage of retrieval was already very pronounced in all groups, it may be speculated that elaborated strategies such as decomposition are used more often in more advanced arithmetic tasks (as can be seen by tendency in the LA and NA groups) and may play a more important role in later stages of elementary school arithmetic. Regarding the high usage of unspecific strategies, Wylie, Jordan, and Mulhern (2012) introduced two new terms to describe unobservable and not described behavior by children's self-report: First, delayed retrieval (no reported or observed strategy and response latency greater than 5 seconds, with the child assumed to be working out the answer in his or her head and relying on working memory during calculation) and second, automatic retrieval (no reported or observed strategy and response within 5 seconds, assumed to involve direct recall of the solution from memory). Both categories differ from the strategies we used in our study but are strongly related to the behavior we coded as unspecific. Since these strategies were rather prevalent in our sample, it is plausible that our category of unspecific strategies may also include both delayed and automatic retrieval.

To conclude, we demonstrated in our study that not only children with LA differ from NA children. In addition, we demonstrated that also children with HA exhibit a unique pattern of strategy behavior in early mathematics.

\section{Acknowledgements}

We would like to thank all the participating children. Moreover, we are grateful to our student assistants for their help with data collection and analysis. This research was funded by the Hessian initiative for the development of scientific and economic excellence (LOEWE).

\section{References}

Canobi, K. H. (2004). Individual differences in children's addition and subtraction. Cognitive Development, 19, 81-93. http://dx.doi.org/10.1016/j.cogdev.2003.10.001 
Canobi, K. H., Reeve, R. A., \& Pattison, P. E. (2003). Patterns of knowledge in children's addition. Developmental Psychology, 39, 521-534. http://dx.doi.org/10.1037/0012-1649.39.3.521

Geary, D. C., Brown, S. C., \& Samaranayake, V. A. (1991). Cognitive addition: A short longitudinal study of strategy choice and speed-of-processing differences in normal and mathematically disabled children. Developmental Psychology, 27, 787-797. http://dx.doi.org/10.1037/0012-1649.27.5.787

Geary, D. C., Hamson, C., \& Hoard, M. (2000). Numerical and arithmetical cognition: A longitudinal study of process and concept deficits in children with learning disability. Journal of Experimental Child Psychology, 77, 236-263. http://dx.doi.org/10.1006/jecp.2000.2561

Geary, D. C., \& Hoard, M. K. (2005). Learning disabilities in arithmetic and mathematics: Theoretical and empirical perspectives. In J. I. D. Campbell (Ed.), Handbook of mathematical cognition (pp. 253-267). New York: Psychology Press.

Geary, D. C., Hoard, M., Nugent, L., \& Byrd Craven, J. (2007). Strategy use, long-term memory, and working memory capacity. In D. Berch, \& M. Mazzocco (Eds.), Why is math so hard for some children? (pp. 83-105). Baltimore, MD: Paul H. Brookes.

Geary, D. C., Bailey, D. H., Littlefield, A., Wood, P., Hoard, M. K., \& Nugent, L. (2009). First-grade predictors of mathematical learning disability: A latent class trajectory analysis. Cognitive Development, 24, 411-429. http://dx.doi.org/10.1016/j.cogdev.2009.10.001

Greer, T., \& Dunlap, W. P. (1997). Analysis of variance with ipsative measures. Psychological Methods, 2, 200-207. http://dx.doi.org/10.1037/1082-989X.2.2.200

Grube, D., Weberschock, U., Baum, M., \& Hasselhorn, M. (2010). Diagnostisches Inventar zu Rechenfertigkeiten im Grundschulalter (DIRG). Göttingen: Hogrefe. [Diagnostic inventory on calculation abilities in elementary-school aged children]

Imbo, I., \& Vandierendonck, A. (2007). The development of strategy use in elementary school children: Working memory and individual differences. Journal of Experimental Child Psychology, 96, 284-309. http://dx.doi.org/10.1016/j.jecp.2006.09.001

Jordan, N. C., Kaplan, D., Ramineni, C., \& Locuniak, M. N. (2008). Development of number combination skill in the early school years: When do fingers help? Developmental Science, 11, 662-668. http://dx.doi.org/10.1111/j.1467-7687.2008.00715.x

Lemaire, P., \& Siegler, R. S. (1995). Four aspects of strategic change: Contributions to children's learning of multiplication. Journal of Experimental Psychology: General, 124, 83-97. http://dx.doi.org/10.1037/0096-3445.124.1.83

Lemaire, P., \& Calliès, S. (2009). Children's strategies in complex arithmetic. Journal of Experimental Child Psychology, 103, 49-65. http://dx.doi.org/10.1016/j.jecp.2008.09.007

Lindberg, S., Lonnemann, J., Linkersdörfer, J., Biermeyer, E., Mähler, C., Hasselhorn, M., \& Lehmann, M. (2011). Early strategies of elementary school children's single word reading. Journal of Neurolinguistics, 24 , 556-570. http://dx.doi.org/10.1016/j.jneuroling.2011.02.003

Mabbott, D. J., \& Bisanz, J. (2003). Developmental change and individual differences in children's multiplication. Child Development, 74, 1091-1107. http://dx.doi.org/10.1111/1467-8624.00594

Miller, G. A. (2000). Wörter. Streifzüge durch die Psycholinguistik. Heidelberg: Spektrum.

Ostad, S. A. (1997). Developmental differences in addition strategies: A comparison of mathematically disabled and mathematically normal children. British Journal of Educational Psychology, 67, 345-357. http://dx.doi.org/10.1111/j.2044-8279.1997.tb01249.x

Rittle-johnson, B., \& Siegler, R. S. (1999). Learning to spell: Variability, choice, and change in children's strategy use. Child Development, 70, 332-348. http://dx.doi.org/10.1111/1467-8624.00025

Siegler, R. S. (1988). Strategy choice procedures and the development of multiplication skills. Journal of Experimental Psychology: General, 117, 258-275. http://dx.doi.org/10.1037/0096-3445.117.3.258

Siegler, R. (1996). Emerging minds: The process of change in children's thinking. New York: Oxford University Press.

Siegler, R. S. (2007). Cognitive variability. Developmental Science, 10, 104-109. http://dx.doi.org/10.1111/j.1467-7687.2007.00571.x 
Siegler, R. S. (2006). Microgenetic analyses of learning. In D. Kuhn, \& R. S. Siegler (Eds.), Handbook of child psychology: Volume 2: Cognition, perception, and language (6th ed., pp. 464-510). Hoboken, NJ: Wiley.

Siegler, R., \& Shrager, J. (1984). Strategy choice in addition and subtraction: How do children know what to do? In C. Sophian (Ed.), Origins of cognitive skills. Hillsdale, NJ: Lawrence Erlbaum.

Wylie, J., Jordan, J.-A., \& Mulhern, G. (2012). Strategic development in exact calculation: Group and individual differences in four achievement subtypes. Journal of experimental child psychology, 113(1), 112-30. http://dx.doi.org/10.1016/j.jecp.2012.05.005

Zbrodoff, N. J., \& Logan, G. D. (2005). What everyone finds: The problem size effect. In J. I. D. Campbell (Ed.), Handbook of mathematical cognition (pp. 331-345). New York: Psychology Press. 